$\mathrm{PM} / 95-39$

CPT-95/P.3247

September 1995

\title{
Anomalous $Z^{\prime}$ effects in the WW channel at NLC
}

\author{
P. Chiappetta ${ }^{a}$, F.M. Renard ${ }^{b}$ and C. Verzegnassi ${ }^{c}$ \\ ${ }^{a}$ Centre de Physique Théorique, UPR 7061, \\ CNRS Luminy, Case 907, F-13288 Marseille Cedex 9. \\ ${ }^{b}$ Physique Mathématique et Théorique, CNRS-URA 768, \\ Université de Montpellier II, F-34095 Montpellier Cedex 5. \\ ${ }^{c}$ Dipartimento di Fisica, Università di Lecce \\ CP193 Via Arnesano, I-73100 Lecce, \\ and INFN, Sezione di Lecce, Italy.
}

\begin{abstract}
We consider the virtual signals of a $Z^{\prime}$ of very general type in the process $e^{+} e^{-} \rightarrow$ $W^{+} W^{-}$at a future linear collider (NLC). We show that possible deviations from the SM predictions in this channel are related to similar deviations in the purely leptonic one in a way that is only characteristic of this $Z^{\prime}$ model, and not in general of possible competitor models with anomalous gauge couplings.
\end{abstract}




\section{Introduction}

The existence of one extra $Z\left(\equiv Z^{\prime}\right)$, with a mass not far from the conventional electroweak breaking scale of a few hundred $\mathrm{GeV}$, has been naturally expected in several, theoretically appealing, models whose motivations vary from those of the "historical" $S O(10), E_{6}$ proposals [1] to those of a number of more recent publications [2]. Owing to the lack of theoretical predictions on the mass of this extra $Z\left(\equiv M_{Z^{\prime}}\right)$, both direct production and virtual signals have been considered in the literature. In particular, from various analyses on $Z$ resonance [3] (where, strictly speaking, no information can be derived on $M_{Z^{\prime}}$, with the remarkable exception of a very small number of "constrained" models) the conclusion emerges that the $Z^{\prime}$ mixing with $Z$ can be ignored for all theoretical analyses at higher energy $e^{+} e^{-}$colliders where either final fermionic channels [4] or $W W$ channel with no initial lepton polarization [5] are considered. Direct production at present and future colliders has also been examined in several papers [6]. This might certainly be relevant for hadronic colliders i.e. for Tevatron and LHC. Since the present experimental limits are already of the order of five hundred GeV [7], only virtual effects have some interest at the future $e^{+} e^{-}$colliders, in particular at LEP2 and at a $500 \mathrm{GeV}$ linear collider (NLC) [8], that we shall consider in this paper as the realistic possibility of a not too far future. In particular, a previous realistic theoretical analysis of virtual effects in the fermionic final channel at NLC that included QED effects has been performed for the specific case of an $E_{6}$ generated $Z^{\prime}$ [9]. The aim of this short paper is that of presenting a preliminary generalization of the analysis of ref. [9] which treats the $Z^{\prime}$ couplings to fermions as free parameters (assuming charged lepton universality) and also considers the possibility of a final $W W$ state, without initial lepton polarization. As we shall show, the combination of the $W W$ channel with the purely leptonic ones would evidentiate special correlations between the different effects that would be an intrinsic characteristics of any model with such a general $Z^{\prime}$.

Following our previous remarks on the $Z-Z^{\prime}$ mixing, the virtual effects of a $Z^{\prime}$ in the process of $e^{+} e^{-}$annihilation into leptons or $W$ pairs at c.m. squared energy $\equiv q^{2}$ can be described, at tree level, by adding to the Standard Model $\gamma, Z$ and $\nu$ exchanges the diagram with $Z^{\prime}$ exchange. The overall effect in the relevant scattering amplitudes will be summarized by the following expressions:

$$
\begin{gathered}
A_{l l}^{(0)}\left(q^{2}\right)=A_{l l}^{(0)(\gamma, Z)}\left(q^{2}\right)+A_{l l}^{(0)\left(Z^{\prime}\right)}\left(q^{2}\right) \\
A_{l W}^{(0)}\left(q^{2}\right)=A_{l W}^{(0)(\gamma, Z, \nu)}\left(q^{2}\right)+A_{l W}^{(0)\left(Z^{\prime}\right)}\left(q^{2}\right)
\end{gathered}
$$

where $l=e, \mu, \tau$ and we assume universal $Z^{\prime} l l$ couplings. In eq.(1) one should read:

$$
\begin{gathered}
A_{l l}^{(0)(\gamma)}\left(q^{2}\right)=\frac{i e_{0}^{2}}{q^{2}} \bar{v}_{l} \gamma_{\mu} u_{l} \bar{u}_{l} \gamma^{\mu} v_{l} \\
A_{l l}^{(0)(Z)}\left(q^{2}\right)=\frac{i}{q^{2}-M_{0 Z}^{2}}\left(\frac{g_{0}^{2}}{4 c_{0}^{2}}\right) \bar{v}_{l} \gamma_{\mu}\left(g_{V l}^{(0)}-\gamma_{5} g_{A l}^{(0)}\right) u_{l} \bar{u}_{l} \gamma^{\mu}\left(g_{V l}^{(0)}-\gamma_{5} g_{A l}^{(0)}\right) v_{l}
\end{gathered}
$$




$$
A_{l l}^{(0)\left(Z^{\prime}\right)}\left(q^{2}\right)=\frac{i}{q^{2}-M_{0 Z^{\prime}}^{2}}\left(\frac{g_{0}^{2}}{4 c_{0}^{2}}\right) \bar{v}_{l} \gamma_{\mu}\left(g_{V l}^{\prime(0)}-\gamma_{5} g_{A l}^{\prime(0)}\right) u_{l} \bar{u}_{l} \gamma^{\mu}\left(g_{V l}^{\prime(0)}-\gamma_{5} g_{A l}^{(0)}\right) v_{l}
$$

$\left(e_{0}^{2}=g_{0}^{2} s_{0}^{2}, s_{0}^{2}=1-c_{0}^{2}\right)$. Note the choice of normalization in eq.(4): $g_{A l}^{(0)}=-\frac{1}{2}$ and $\left.g_{V l}^{(0)}=g_{A l}^{(0)}-2 Q_{l} s_{0}^{2}\right)$.

Analogous expressions can be easily derived for eq.(2). We shall only give here the relevant $Z^{\prime}$ contribution, which reads:

$$
A_{l W}^{(0)\left(Z^{\prime}\right)}\left(q^{2}\right)=\frac{i}{q^{2}-M_{0 Z^{\prime}}^{2}}\left(\frac{g_{0}}{2 c_{0}}\right) \bar{l}_{l} \gamma^{\mu}\left(g_{V l}^{\prime(0)}-\gamma_{5} g_{A l}^{\prime(0)}\right) u_{l} e_{0} g_{Z^{\prime} W W} P_{\alpha \beta \mu} \epsilon_{\alpha}^{*}\left(p_{1}\right) \epsilon_{\beta}^{*}\left(p_{2}\right)
$$

where

$$
P_{\alpha \beta \mu}=g_{\mu \beta}\left(2 p_{2}+p_{1}\right)_{\alpha}+g_{\beta \alpha}\left(p_{1}-p_{2}\right)_{\mu}-g_{\mu \alpha}\left(2 p_{1}+p_{2}\right)_{\beta}
$$

and $p_{1,2}$ are the four-momenta of the outgoing $W^{+,-}$. In this expression we have assumed that the $Z^{\prime} W W$ vertex has the usual Yang-Mills form. We do not consider the possibility of anomalous magnetic or quadrupole type of couplings. In fact in most of the popular examples based on extended gauge models [10], even with a strong coupling regime [11], or in compositeness inspired schemes [12], only the Yang-Mills form appears or at least dominates over anomalous forms in the large $M_{Z^{\prime}} / M_{Z}$ limit (there are however exceptions, see for example [13]). An analysis with anomalous $Z W W$ and $Z^{\prime} W W$ coupling forms is possible along the lines of ref. [14] but is beyond the scope of this paper. Our analysis will be nevertheless rather general as the trilinear $Z^{\prime} W W$ coupling $g_{Z^{\prime} W W}$ will be treated as a free parameter, not necessarily proportional to the $Z-Z^{\prime}$ mixing angle as for example it would happen in a "conventional" $E_{6}$ picture.

For the purposes of this paper, it will be particularly convenient to describe the virtual $Z^{\prime}$ effect as an "effective" modification of the $\underline{Z}$ and $\gamma$ couplings to fermions and $W$ pairs. As one can easily derive, this corresponds to the use of the following "modified" leptonic $\gamma, Z$ couplings (denoted in the following with a star) to describe the $e^{+} e^{-} \rightarrow l^{+} l^{-}$process:

$$
\begin{gathered}
e_{0}^{*}=e_{0}\left[1-\frac{q^{2}}{M_{Z^{\prime}}^{2}-q^{2}}\left(\frac{g_{V l}^{(0)}}{4 s_{0}^{2} c_{0}^{2}}\right)\left(\xi_{V l}-\xi_{A l}\right)^{2}\right]^{1 / 2} \\
g_{A l}^{*(0)}=g_{A l}^{(0)}\left[1-\frac{q^{2}-M_{Z}^{2}}{M_{Z^{\prime}}^{2}-q^{2}} \xi_{A l}^{2}\right]^{1 / 2} \\
g_{V l}^{*(0)}=g_{V l}^{(0)}\left[1-\frac{q^{2}-M_{Z}^{2}}{M_{Z^{\prime}}^{2}-q^{2}} \xi_{A l}\left(\xi_{V l}-\xi_{A l}\right)\right] \cdot\left[1-\frac{q^{2}-M_{Z}^{2}}{M_{Z^{\prime}}^{2}-q^{2}} \xi_{A l}^{2}\right]^{1 / 2}
\end{gathered}
$$

and to the use of the following modified trilinear couplings that fully describe the effect in the final $e^{+} e^{-} \rightarrow W W$ process (without modifying the initial $\gamma, Z$ leptonic couplings):

$$
g_{\gamma W W}^{*(0)}=g_{\gamma W W}^{(0)}+g_{Z^{\prime} W W}^{(0)} \frac{q^{2}}{M_{Z^{\prime}}^{2}-q^{2}} g_{V l}^{(0)}\left(\xi_{V l}-\xi_{A l}\right)
$$




$$
g_{Z W W}^{*(0)}=g_{Z W W}^{(0)}-g_{Z^{\prime} W W}^{(0)} \frac{q^{2}-M_{Z}^{2}}{M_{Z^{\prime}}^{2}-q^{2}} \xi_{A l}
$$

In the previous equations, the following definitions have been used:

$$
\begin{aligned}
& \xi_{V l}=g_{V l}^{\prime(0)} / g_{V l}^{(0)} \\
& \xi_{A l}=g_{A l}^{\prime(0)} / g_{A l}^{(0)}
\end{aligned}
$$

Our normalization is such that:

$$
\begin{gathered}
g_{\gamma W W}^{(0)}=1 \\
g_{Z W W}^{(0)}=c_{0} / s_{0}
\end{gathered}
$$

Note that, strictly speaking, only bare quantities should appear in the previous equations. In practice, however, we shall treat the $Z^{\prime}$ effect on the various observables at one loop in the $(\gamma, Z)$ Standard Model sector, and in an "effective" tree level for what concerns the $Z^{\prime}$ parameters. As a result of this (standard) approach, whose validity is obviously related to the (implicit) assumption that $M_{Z^{\prime}}^{2}$ is "large" at the energy scale of the experiment, only the physical $\gamma, Z$ parameters, the "physical" $Z^{\prime}$ mass and the "physical" $Z$ ' couplings will remain in the various theoretical expressions. Note that the definition of "physical" $Z^{\prime}$ couplings is plagued with an intrinsic ambiguity that would only be solved once this particle were discovered and its decays measured. This will not represent a problem in our approach, as we shall see, since our philosophy will rather be that of calculating functional relationships between different (experimentally measurable) $Z^{\prime}$ shifts. In this spirit, we shall use from now on only notations without "bare" indices on the various parameters.

The basic idea of our approach is provided by the observation that the modified trilinear gauge couplings eqs.(11),(12) contain the same combinations of modified fermionic $\gamma, Z$ couplings eqs.(8-10) that would appear in the leptonic final state, with only one extra free parameter i.e. the $Z^{\prime} W W$ coupling $g_{Z^{\prime} W W}$. This implies that it must be possible to find a precise relationship between the two modified trilinear gauge couplings eqs.(11),(12) and some set of leptonic observables by simply eliminating the free parameter $g_{Z^{\prime} W W}$ in these expressions. To fully understand what type of relationships will emerge, it is oportune to write down at this point the expression of the $Z^{\prime}$ effects in the two leptonic observables that will certainly be measured to a very good accuracy at NLC, i.e. the muon (三 lepton) cross section $\sigma_{\mu}\left(q^{2}\right)$ and the muon forward-bacward asymmetry $A_{F B, \mu}\left(q^{2}\right)$. To calculate these expressions is straightforward and the rigorous derivation has been already performed in previous papers, to which we refer for a detailed discusssion [15]. Here we shall only show for sake of self-completeness an approximate procedure where only the numerically relevant terms are retained. For this aim it will be sufficient to start from the following expressions of $\sigma_{\mu}, A_{F B, \mu}$ at Born level without the $Z^{\prime}$ contribution: 


$$
\sigma_{\mu}^{(0)}\left(q^{2}\right)=\frac{q^{2}}{12 \pi}\left[\left(\frac{e_{0}^{2}}{q^{2}}\right)^{2}+\frac{1}{\left(q^{2}-M_{0 Z}^{2}\right)^{2}}\left(\frac{g_{0}^{2}}{4 c_{0}^{2}}\right)\left(g_{V l}^{(0) 2}+g_{A l}^{(0) 2}\right)^{2}\right]
$$

(we have omitted the $\gamma-Z$ interference that is numerically negligible [15]).

$$
A_{F B, \mu}^{(0)}\left(q^{2}\right)=\frac{\pi q^{2}}{\sigma_{\mu}\left(q^{2}\right)}\left[\frac{1}{\left(q^{2}-M_{0 Z}^{2}\right)^{2}}\left(\frac{1}{4 \pi^{2}}\right)\left(\frac{g_{0}^{2}}{4 c_{0}^{2}}\right)^{2} g_{V l}^{(0) 2} g_{A l}^{(0) 2}+\frac{1}{q^{2}\left(q^{2}-M_{0 Z}^{2}\right)}\left(\frac{1}{8 \pi^{2}}\right)\left(\frac{g_{0}^{2}}{4 c_{0}^{2}}\right) e_{0}^{2} g_{A l}^{(0) 2}\right]
$$

The prescription for deriving the $Z^{\prime}$ effect is now the following. One replaces the quantities $e_{0}, g_{A l}^{(0)}, g_{V l}^{(0)}$ that appear in eqs.(17),(18) by the starred ones given in eqs.(8)-(10). All the remaining bare $(\gamma, Z)$ parameters will then be replaced by the known Standard Model expressions valid at one loop, that will contain certain "physical" quantities and certain one-loop "corrections". For the purposes of this paper, where only the (small) $Z^{\prime}$ effects are considered, the latter corrections can be ignored and one can write the relevant shifts in terms of $Z^{\prime}$ parameters and of $\gamma, Z$ "physical" quantities (more precisely, as one can guess, $\alpha(0)$ and $s_{E f f}^{2}\left(M_{Z}^{2}\right)$ ), and for a more detailed discussion we refer to ref. [15].

Defining the relative $Z^{\prime}$ shifts as :

$$
\frac{\delta \sigma_{\mu}^{\left(Z^{\prime}\right)}}{\sigma_{\mu}}=\frac{\sigma_{\mu}^{\left(\gamma, Z, Z^{\prime}\right)}-\sigma_{\mu}^{(\gamma, Z)}}{\sigma_{\mu}^{(\gamma, Z)}}
$$

(and an analogous definition for the asymmetry),

it is now relatively simple to derive the expressions:

$$
\begin{gathered}
\frac{\delta \sigma_{\mu}}{\sigma_{\mu}}=\frac{2}{\kappa^{2}\left(q^{2}-M_{Z}^{2}\right)^{2}+q^{4}}\left[\kappa^{2}\left(q^{2}-M_{Z}^{2}\right)^{2} \tilde{\Delta}^{\left(Z^{\prime}\right)} \alpha\left(q^{2}\right)-q^{4}\left(R^{\left(Z^{\prime}\right)}\left(q^{2}\right)+\frac{1}{2} V^{\left(Z^{\prime}\right)}\left(q^{2}\right)\right)\right] \\
\left.\frac{\delta A_{F B, \mu}}{A_{F B, \mu}}=\frac{q^{4}-\kappa^{2}\left(q^{2}-M_{Z}^{2}\right)^{2}}{\kappa^{2}\left(q^{2}-M_{Z}^{2}\right)^{2}+q^{4}}\left[\tilde{\Delta}^{\left(Z^{\prime}\right)} \alpha\left(q^{2}\right)+R^{\left(Z^{\prime}\right)}\left(q^{2}\right)\right]+\frac{q^{4}}{\kappa^{2}\left(q^{2}-M_{Z}^{2}\right)^{2}+q^{4}} V^{\left(Z^{\prime}\right)}\left(q^{2}\right)\right]
\end{gathered}
$$

with (using the same notations as in ref.[15]):

$$
\begin{gathered}
\tilde{\Delta}^{\left(Z^{\prime}\right)} \alpha\left(q^{2}\right)=\frac{q^{2}}{q^{2}-M_{Z^{\prime}}^{2}}\left(\frac{v_{1}^{2}}{16 s_{1}^{2} c_{1}^{2}}\right)\left(\xi_{V l}-\xi_{A l}\right)^{2} \\
R^{\left(Z^{\prime}\right)}\left(q^{2}\right)=\left(\frac{q^{2}-M_{Z}^{2}}{M_{Z^{\prime}}^{2}-q^{2}}\right) \xi_{A l}^{2} \\
V^{\left(Z^{\prime}\right)}\left(q^{2}\right)=\left(\frac{q^{2}-M_{Z}^{2}}{M_{Z^{\prime}}^{2}-q^{2}}\right)\left(\frac{v_{1}}{4 s_{1} c_{1}}\right) \xi_{A l}\left(\xi_{V l}-\xi_{A l}\right)
\end{gathered}
$$

where 


$$
\kappa=\frac{\alpha M_{Z}}{9 \Gamma_{l}}
$$

and $v_{1}=-2 g_{V l}=1-4 s_{1}^{2} ; s_{1}^{2} c_{1}^{2}=\frac{\pi \alpha}{\sqrt{2} G_{\mu} M_{Z}^{2}}$.

As one sees from the previous equations, the $Z^{\prime}$ effects can be expressed by certain quadratic expressions of the parameters $\xi_{A l},\left(\xi_{V l}-\xi_{A l}\right)$. A much simpler dependence is exhibited by the modified trilinear couplings, as one sees from eqs.(11),(12). Adopting the notations that are found in recent literature [16], [14], we find for the $Z^{\prime}$ effect in this case

$$
\begin{gathered}
\delta_{\gamma}^{\left(Z^{\prime}\right)} \equiv g_{\gamma W W}^{*}-1=g_{Z^{\prime} W W}\left(\frac{q^{2}}{M_{Z^{\prime}}^{2}-q^{2}}\right) g_{V l}\left(\xi_{V l}-\xi_{A l}\right) \\
\delta_{Z}^{\left(Z^{\prime}\right)} \equiv g_{Z W W}^{*}-\cot \theta_{W}=-g_{Z^{\prime} W W}\left(\frac{q^{2}-M_{Z}^{2}}{M_{Z^{\prime}}^{2}-q^{2}}\right) \xi_{A l}
\end{gathered}
$$

From eqs.(23),(24) one can derive the following constraint:

$$
\delta_{\gamma}^{\left(Z^{\prime}\right)}=\operatorname{tg} \theta_{A} \delta_{Z}^{\left(Z^{\prime}\right)}
$$

where

$$
\operatorname{tg} \theta_{A}=\frac{-q^{2}}{q^{2}-M_{Z}^{2}}\left(\frac{\xi_{V l}-\xi_{A l}}{\xi_{A l}}\right) g_{V l}
$$

A few comments are appropriate at this point. In this description both $g_{\gamma W W}$ and $g_{Z W W}$ couplings are modified by form factor effects whose scale is $M_{Z^{\prime}}$. They identically vanish for $q^{2}=0$ and $q^{2}=M_{Z}^{2}$ respectively. The vanishing of $\delta_{\gamma}$ at $q^{2}=0$ is absolutely required by conservation of electric charge. We then notice that the virtual effect of a general $Z^{\prime}$ in the $W W$ channel is, at first sight, quite similar to that of a possible model with anomalous gauge couplings, that would also produce shifts $\delta_{\gamma}, \delta_{Z} \underline{\text { both }}$ in the $\gamma W W$ and in the $Z W W$ couplings (in the conventional description of anomalous gauge boson couplings the appearence of both $\delta_{\gamma}$ and $\delta_{Z}$ type is rather unusual[14], but can be described using effective lagrangians with $\operatorname{dim}=6$ and $\operatorname{dim}=8$ operators [16]). For this reason, we have called such effects "anomalous" $Z^{\prime}$ effects. But the $Z^{\prime}$ shifts satisfy in fact the constraint given by eq.(28), that corresponds to a certain line in the $\left(\delta_{\gamma}, \delta_{Z}\right)$ plane whose angular coefficient is fixed by the model i.e. by the values of $\xi_{A},\left(\xi_{V}-\xi_{A}\right)$. For example:

$$
\operatorname{tg} \theta_{A}=\frac{-q^{2}}{q^{2}-M_{Z}^{2}}\left[\frac{v_{1}}{2}+\frac{\cos \beta}{\sqrt{\frac{5}{3}} \sin \beta+\cos \beta}\right]
$$

in $E_{6}$ models $(-1<\cos \beta<+1)$,

$$
\operatorname{tg} \theta_{A}=\frac{-q^{2}}{q^{2}-M_{Z}^{2}}\left[\frac{v_{1}}{2}-\frac{2}{\alpha_{R L}}\left(\frac{1}{2 \alpha_{R L}}-\frac{\alpha_{R L}}{4}\right)\right]
$$


in Right-Left symmetric models $\left(\sqrt{\frac{2}{3}}<\alpha_{R L}<\sqrt{2}\right)$,

$$
\operatorname{tg} \theta_{A}=\frac{-q^{2}}{q^{2}-M_{Z}^{2}} \cot \theta_{W}
$$

in $Y$ models,

$$
\operatorname{tg} \theta_{A}=\frac{q^{2}}{q^{2}-M_{Z}^{2}} \cot \theta_{W}\left(\frac{s_{1}^{2}-\lambda_{Y}^{2}}{1-s_{1}^{2}+\lambda_{Y}^{2}}\right)
$$

in $Y_{L}$ models $\left(0<\lambda_{Y}^{2}<1-s_{1}^{2}\right)$.

The values of $\xi_{A},\left(\xi_{V}-\xi_{A}\right)$ are, in turn, directly responsible for deviations in the leptonic channel that would affect $\sigma_{\mu}$ and $A_{F B, \mu}$. This means that a precise functional relationship will exist between the value of $\operatorname{tg} \theta_{A}$ defined by eq.(26) and those of the shifts $\delta \sigma_{\mu}, \delta A_{F B, \mu}$ defined by eqs.(20),(21) that would correspond to a certain surface in the 3$\operatorname{dim}\left(\operatorname{tg} \theta_{A}, \delta^{\left(Z^{\prime}\right)} \sigma_{\mu} / \sigma_{\mu}, \delta^{\left(Z^{\prime}\right)} A_{F B, \mu} / A_{F B, \mu}\right)$ space. To draw this surface requires a dedicated numerical analysis that carefully takes into account the QED initial radiation and the precise experimental set up, which is beyond the purposes of this short paper. Here we shall only illustrate, with a couple of particularly simple examples, what would be typical signatures of this type of $Z^{\prime}$ effects.

We begin with the case [17 of a $Z^{\prime}$ whose couplings to the fermions are "essentially" the same as those of the Standard Model $Z$ (this is usually called the "standard $Z$ ' model"), leaving the $Z^{\prime} W W$ coupling free. In this case $\delta_{\gamma}^{\left(Z^{\prime}\right)}=0$ and $\delta_{Z}^{\left(Z^{\prime}\right)}$ is given by eq.(24) with $\xi_{A l}$ of order one. Eqs.(19),(20) become now at $500 \mathrm{GeV}$ :

$$
\begin{gathered}
\frac{\delta \sigma_{\mu}^{\left(Z^{\prime}\right)}}{\sigma_{\mu}}\left(\xi_{V l}=\xi_{A l}\right) \simeq-0.234 \frac{q^{2}-M_{Z}^{2}}{M_{Z^{\prime}}^{2}-q^{2}} \xi_{A l}^{2} \\
\frac{\delta^{\left(Z^{\prime}\right)} A_{F B, \mu}}{A_{F B, \mu}}\left(\xi_{V l}=\xi_{A l}\right) \simeq-0.735 \frac{q^{2}-M_{Z}^{2}}{M_{Z^{\prime}}^{2}-q^{2}} \xi_{A l}^{2}
\end{gathered}
$$

showing that, for this situation, the asymmetry is more sensitive to the effect. In terms of the asymmetry we have now:

$$
\delta_{Z}^{\left(Z^{\prime}\right)}\left(\xi_{V l}=\xi_{A l}\right) \simeq\left(\frac{g_{Z^{\prime} W W}}{0.735 \xi_{A l}}\right) \frac{\delta^{\left(Z^{\prime}\right)} A_{F B, \mu}}{A_{F B, \mu}}=-g_{Z^{\prime} W W} \frac{q^{2}-M_{Z}^{2}}{M_{Z^{\prime}}^{2}-q^{2}} \xi_{A l}
$$

We shall now introduce the following ansatz concerning the theoretical expressions of $g_{Z^{\prime} W W}$, that we shall write as:

$$
g_{Z^{\prime} W W}=\left[c \frac{M_{Z}^{2}}{M_{Z^{\prime}}^{2}}\right] \operatorname{cotg} \theta_{W}
$$

The constant $c$ would be of order one for the "conventional" models [10 where the $Z^{\prime}$ couples to $\mathrm{W}$ only via the $Z-Z^{\prime}$ mixing (essentially contained in the bracket). But for a general model, $c$ could be larger, as one can see for some special cases of composite 
models, for example with excited $Z^{*}$ states [13 or when the $Z^{\prime}$ participates in a strong coupling regime [11].

In fact, a stringent bound on $c$ comes from the request that the $Z^{\prime}$ width into $W W$ is "small" compared to the $Z^{\prime}$ mass. Inposing the (reasonable) limit

$$
\Gamma_{Z^{\prime} W W} \lesssim \frac{1}{10} M_{Z^{\prime}}
$$

leads to the condition

$$
c \lesssim 10
$$

and this will be our very general working assumption.

Using eq.(37) we can rewrite the $Z^{\prime}$ effect as

$$
\delta_{Z}^{\left(Z^{\prime}\right)}\left(\xi_{V l}=\xi_{A l}\right)=-\xi_{A l} \frac{q^{2}-M_{Z}^{2}}{M_{Z^{\prime}}^{2}-q^{2}} c\left(\frac{M_{Z}^{2}}{M_{Z^{\prime}}^{2}}\right) \cot \theta_{W}
$$

For $\xi_{A l} \simeq 1$, at the NLC energy the detectability request [14], $\left|\delta_{Z}\right| \gtrsim 10^{-2}$ corresponds to the condition:

$$
c \gtrsim 2 \times 10^{-4} \frac{M_{Z^{\prime}}^{2}}{M_{Z}^{2}}\left[\frac{M_{Z^{\prime}}^{2}-(500 G e V)^{2}}{M_{Z}^{2}}\right]
$$

which obeys the constraint eq.(32) for all values of $M_{Z^{\prime}}$ such that

$$
M_{Z^{\prime}} \lesssim 1.4 T e V
$$

and, for the limiting value $M_{Z^{\prime}}=1.4 T e V$, a reasonable relative shift of approximately ten percent in the asymmetry would be produced, that would not be missed at the expected experimental accuracy.

As a second example, we consider the orthogonal case $\xi_{A l}=0$ for "large" values of $\xi_{V l}$ (say, $\left.\xi_{V l} \simeq 10\right)$. Such an order of magnitude corresponds to several "conventional" models. For example in $E_{6}$

$$
\xi_{V l}=-\frac{4 s_{1} \cos \beta}{v_{1}}
$$

reaches $\xi_{V l} \simeq 5$ for the $\chi$ model $(\cos \beta=1)$, and for a $Y$ model

$$
\xi_{V l}=\frac{3 s_{1} c_{1}}{v_{1} \lambda_{Y}}\left(1-\frac{\lambda_{Y}^{2}}{1-s_{1}^{2}}\right)^{1 / 2}
$$

reaches $\xi_{V l} \simeq 12$ for $\lambda_{Y}^{2}=s_{1}^{2}$. In this situation, $\delta_{Z}^{\left(Z^{\prime}\right)}=0$, and

$$
\begin{gathered}
\frac{\delta \sigma_{\mu}^{\left(Z^{\prime}\right)}}{\sigma_{\mu}}\left(\xi_{A l}=0\right) \simeq-1.11 \times 10^{-2} \frac{q^{2}}{M_{Z^{\prime}}^{2}-q^{2}} \xi_{V l}^{2} \\
\frac{\delta^{\left(Z^{\prime}\right)} A_{F B, \mu}}{A_{F B, \mu}} \simeq 4.71 \times 10^{-3} \frac{q^{2}}{M_{Z^{\prime}}^{2}-q^{2}} \xi_{V l}^{2}
\end{gathered}
$$


and one sees that now the sensible quantity is $\sigma_{\mu}$. For the trilinear shift $\delta_{\gamma}^{\left(Z^{\prime}\right)}$ we have :

$$
\delta_{\gamma}^{\left(Z^{\prime}\right)}\left(\xi_{A l}=0\right) \simeq g_{V l} \xi_{V l} \frac{q^{2}}{M_{Z^{\prime}}^{2}-q^{2}} c\left(\frac{M_{Z}^{2}}{M_{Z^{\prime}}^{2}}\right) \cot \theta_{W}
$$

In correspondence to the limiting value $c=10$ and for $M_{Z^{\prime}}=1.4 T e V$, we get for $\xi_{V l}=O(10)$ a value $\delta_{\gamma}^{\left(Z^{\prime}\right)}=O\left(10^{-2}\right)$ i.e. the same size as $\delta_{Z}^{\left(Z^{\prime}\right)}$ in the first example (the relative shift in $\sigma_{\mu}$ would now be of approximately fifteen percent, that would certainly not escape experimental detection). This value should be compared to that predicted by dedicated analyses, that to our knowledge are still missing.

We have also looked whether there are reasonable possibilities to observe such type of $Z^{\prime} W W$ effects at LEP2. Assuming the lowest allowed mass $M_{Z^{\prime}}=600 \mathrm{GeV}$ and the strongest $Z^{\prime} W W$ coupling with $c=10$ in both extreme cases studied above, one gets at most a two percent effect in $\delta_{\gamma}^{\left(Z^{\prime}\right)}$ or $\delta_{Z}^{\left(Z^{\prime}\right)}$ which is below the observability limit expected from ref. 14.

In conclusion, we can summarize the main points of our (preliminary) analysis as follows. A $Z^{\prime}$ belonging to a quite general model, with "reasonable" couplings to $W$ (and to fermions), would produce effects in the $W W$ and in the leptonic channel that would be related in a quite special way and, at least in some simple cases, visible in both channels at a high energy $e^{+} e^{-}$collider. If, at the time of a possible NLC run, models with anomalous gauge couplings will not be ruled out, this fact would certainly be a powerful tool for discrimination. In fact, in the anomalous gauge coupling case, the parameters that affect the $W W$ channel are totally independent of those that affect the leptonic one so that no kind of relationships will in general exist. If, on the contrary, anomalous gauge couplings were out of interest, the constraints that we derived would certainly help to achieve a proper $Z^{\prime}$ identification, in case this particle were actually produced e.g. at the CERN LHC.

\section{Acknowledgements}

This work has been partially supported by the EC contract CHRX-CT94-0579. 


\section{References}

[1] For a review see, J. Hewett and T. Rizzo, Phys. Rep. C183 (1989) 193;

R. N. Mohapatra, Unification and supersymmetries, Berlin, Heidelberg, New York,Springer 1989.

[2] D. Kosower, Phys. Rev. D48 (1993) 1288.

[3] J. Layssac, F.M. Renard and C. Verzegnassi, Phys. Lett. B287 (1992) 267 , Z. Phys. C53 (1992) 97;

E. Nardi, F. Roulet and D. Tommasini, Phys. Rev. D46 (1992) 3040;

P. Langacker and M. Luo, Phys. Rev. D45 (1992) 278;

F. Del Aguila et al, Nucl. Phys. B372 (1992) 13.

[4] A. Leike, S. Riemann and T. Riemann Phys. Lett. B291 (1992) 187.

[5] A. Babich, A. Pankov and N. Paver, Phys. Lett. B299 (1993) 351 ; Phys. Lett. B346 (1995) 303 ;

A. Pankov and N. Paver, Phys. Rev. D48 (1993) 63.

[6] F. Del Aguila, M. Cvetic and P. Langacker, in Proceedings of the Second International Workshop on Physics and Experiments at Linear Colliders, Waikola,Hawai, 1993, vol II p490;

P. Chiappetta, M. Greco et al, in Proceedings of LHC Workshop, Aachen, 1990, CERN-90-10 Vol II, p685.

[7] CDF Collaboration, F. Abe et al., FERMILAB-PUB-94-198-E(1994).

[8] Proc. of the Workshop on $e^{+} e^{-}$Collisions a $500 \mathrm{GeV}$ : The Physics Potential, DESY 92-123A,B,C(1992), ed. P.Zerwas;

A. Leike, Z. Phys. C62 (1994) 265.

[9] A. Leike and T. Riemann, Z. Phys. C51 (1991) 113;

A. Leike, T. Riemann and M. Sachvitz, Phys. Lett. B241 (1990) 267.

[10] F. Del Aguila, M. Quiros and F. Zwirner, Nucl. Phys. B287 (1987) 419.

[11] J.L. Kneur and D. Schildknecht, Nucl. Phys. B357 (357) 1991;

J.L. Kneur, M. Kuroda and D. Schildknecht, Phys. Lett. B262 (93) 1991 ;

M. Bilenky et.al., Phys. Lett. B316 (1993) 345 ;

R. Casalbuoni, S. de Curtis, D. Dominici and R. Gatto, Phys. Lett. B155 (1985) 95 ; Nucl. Phys. B282 (1987) 235. 
[12] M.Kuroda, D.Schildknecht and K.H.Schwarzer, Nucl. Phys. B261 (432) 1985.

[13] U.Baur, D.Schildknecht and K.H.Schwarzer, Phys. Rev. D37 (297) 1987.

[14] M. Bilenky, J.L. Kneur, F.M. Renard and D. Schildknecht, Nucl. Phys. B409 (1993) 22 and B419 (1994) 240.

[15] F.M. Renard and C. Verzegnassi, preprint PM/95-35 (1995).

[16] G.J. Gounaris and F.M. Renard, Z. Phys. C59 (1993) 133.

[17] G. Altarelli, B. Mele and M. Ruiz Altaba, Z. Phys. C45 (1989) 109; erratum Z. Phys. C47 (1990) 676. 